\title{
Electrochemical Activation of Expanded Graphite Electrode for Electrochemical Capacitor
}

\author{
Bok H. Ka and Seung M. Oh ${ }^{*, z}$
}

Department of Chemical and Biological Engineering, and Research Center for Energy Conversion

\& Storage, Seoul National University, Seoul 151-774, Korea

\begin{abstract}
An expanded graphite (e-MCMB, mesocarbon microbeads) having a wider interlayer spacing $\left(d_{002}=0.404 \mathrm{~nm}\right)$ than that of common graphites is prepared by heat-treatment of an oxidized MCMB. When the e-MCMB electrode, which gives a negligible capacitance due to a small surface area, is polarized over a certain onset potential $\left[4.6-4.8 \mathrm{~V}\left(\mathrm{vs} \mathrm{Li} / \mathrm{Li}^{+}\right.\right.$) for positive and $1.3-1.0$ $\mathrm{V}$ for negative direction], it is electrochemically activated to be a high-capacitance positive and negative electrode for electrochemical capacitor. The activation process involves an ion intercalation into the interlayer space to generate ion-accessible sites. The intercalation is evidenced by the presence of a voltage plateau in the charge-discharge profiles, and by the widening of the interlayer distance (by in situ X-ray diffraction study) and concomitant electrode swelling (by electrochemical dilatometry) that occur at the same potential region. The electrochemically activated e-MCMB particles carry slitlike pores of ca. $0.45 \mathrm{~nm}$ in the mean interlayer distance, into which ions very likely enter either bare or with partial solvent shells with a mixed adsorption/ intercalation charge storage behavior. A full cell fabricated with two e-MCMB electrodes delivers a volume specific capacitance of 30-24 F mL $\mathrm{mL}^{-1}$ within 100 cycles for a dry electrode pair at a working voltage of $3.7 \mathrm{~V}$.

(C) 2008 The Electrochemical Society. [DOI: 10.1149/1.2953525] All rights reserved.
\end{abstract}

Manuscript submitted February 12, 2008; revised manuscript received June 9, 2008. Published July 25, 2008.

Until now, electric double-layer capacitors (EDLCs), which deliver a higher rate capability and longer cycle life as compared to the modern secondary batteries, have been used as the energy storage device for memory back-up systems. ${ }^{1-6}$ Recently, the markets for EDLCs have been extended to the higher power and higher energy systems such as hybrid electric vehicles. Energy density of the present EDLC, however, does not meet the market's need. Hence, to exploit such new applications, it is necessary to develop electrode materials having a higher energy density than the conventional ones.

The energy density of EDLC is given by $E=1 / 2 C V^{2}$, where $C$ stands for the capacitance per volume or weight, and $V$ the working voltage. An enlargement in either $C$ or $V$ can thus be the way to achieve a high energy density in EDLCs. ${ }^{3,5,7}$ One way to increase the cell voltage is the use of nonaqueous electrolytes. Normally, the cell voltage of EDLCs employing aqueous electrolytes is below 1.2 $\mathrm{V}$, which can, however, be enlarged up to $3.0 \mathrm{~V}$ by using nonaqueous electrolytes. The other approach to increase the energy density is the employment of high-capacitance electrode materials, which are normally high-surface-area conductive materials as the electric double layer is formed at the electrode/electrolyte interface. ${ }^{8-13}$ Until now, activated carbons have been the most popular choice for EDLC electrode materials because they are electrically conductive and possess a high surface area $\left(1000-2000 \mathrm{~m}^{2} \mathrm{~g}^{-1}\right)$. The critical problem for activated carbons when considered as the electrode material for high-energy density EDLCs is the low gravimetric density due to their amorphous and porous structure. The volume-specific capacitance of EDLC fabricated with two activated carbon electrodes is commonly less than $20 \mathrm{~F} \mathrm{~mL}$. $^{-14-16}$

Many attempts have been made to increase the energy density of EDLCs, which can be categorized into two approaches. One is the replacement of one or both activated carbon electrodes with redoxreaction materials; metal oxides, conducting polymers, or intercalation compounds that are commonly used in lithium secondary cells. ${ }^{17-19}$ They are termed as hybrid or asymmetric capacitors. This modification not only increases the specific capacitance $(C)$, but also extends the working voltage $(V)$. The other approach is the application of electrochemical activation to graphitic or graphitizable carbons. ${ }^{20-25}$ An example is the Nanogate capacitor that was introduced by JEOL Ltd., Japan. According to the reports, the $\mathrm{KOH}-$ activated graphitizable carbon, which delivers a negligible capacitance due to a small surface area $\left(<100 \mathrm{~m}^{2} \mathrm{~g}^{-1}\right)$, is converted to a

* Electrochemical Society Active Member

z E-mail: seungoh@snu.ac.kr high-capacitance material by an electrochemical activation. ${ }^{20,21}$ That is, an ion-accessible surface is generated at the interlayer space as a result of ion/solvent co-intercalation into the interlayer. The capacitor fabricated with two such electrodes shows a much improved energy density with a volume-specific capacitance of 25-30 $\mathrm{F} \mathrm{mL}^{-1}$ for a dry electrode pair and working voltage of 3.5$4.0 \mathrm{~V}$.

Here, we report another graphitic material that can be electrochemically activated by electrode polarization in both positive and negative directions, which is the expanded graphite that was prepared by heat-treatment of oxidized graphites (commonly known as graphite oxides). The oxidized graphites were in turn prepared by oxidizing the pristine mesocarbon microbeads (MCMB) with an oxidizing agent in a strong acid medium. ${ }^{26-30}$ The electrochemical activation behavior at the positive and negative electrode was examined independently using a three-electrode cell configuration. To estimate the energy density of this electrode material, a symmetric cell comprising two expanded MCMB (e-MCMB) electrodes was fabricated and its volume-specific capacitance and working voltage were examined after electrochemical activation.

Another concern in this work, in addition to the introduction of graphitic material that can be electrochemically activated, is to clarify the following points fundamental to the understanding of the electrochemical activation process, but not fully addressed in the previous reports due to a limited publication. ${ }^{20-25}$ The first concern is the microscopic structural change evolved in graphitic carbons that may occur in the electrochemical activation process. The second concern is the charge storage mechanism prevailing after electrochemical activation. It is still undetermined whether charge storage is done by an intercalation process or by a simple adsorption. To address these issues, the charge/discharge voltage profiles, and the variation in the interlayer distance of graphitic materials and concomitant electrode volume change, were examined for an e-MCMB electrode before and after electrochemical activation. A conclusion on these issues has been reached by comparing the results to those obtained with an activated carbon and pristine MCMB electrode. The third concern is the electrode swelling that seems unavoidable with intercalation. From a practical viewpoint, a repeated electrode swelling/contraction is not desired because mechanical stress frequently leads to electrode degradation. The electrode swelling/ contraction upon electrochemical activation on the Nanogate capacitor has been examined by monitoring the cell pressure with a dimensionally fixed setup, but it is not clear if the pressure change is caused solely by the dimensional change of electrodes. ${ }^{20,21}$ Rather, it is very likely that gas evolution obscures the electrode swelling/ contraction. In this work, electrochemical dilatometry was utilized 
to trace the electrode swelling/contraction, which is free from the interference caused by gas evolution. The final concern is the nature of prerequisites for graphitic materials to be electrochemically activated. This issue will be discussed by reviewing the literature and providing our own results.

\section{Experimental}

In this work, the MCMB-10-28 (average particle size $=10 \mu \mathrm{m}$, graphitization temperature $=2800^{\circ} \mathrm{C}$, Osaka Gas Co., Japan) was used as the graphite source. The expanded graphite (eMCMB) was prepared by a heat-treatment of oxidized graphite (o$\mathrm{MCMB}$ ) at $250^{\circ} \mathrm{C}$ for $24 \mathrm{~h}$ in Ar atmosphere. The o-MCMB was in turn prepared by oxidizing the MCMB-10-28 with a mixture of strong oxidant and strong acid. In detail, the MCMB-10-28 powder $(5.0 \mathrm{~g})$ and $\mathrm{KMnO}_{4}(10.0 \mathrm{~g})$ were added into a mixture of $95 \%$ sulfuric acid $(60 \mathrm{~mL})$ and $60 \%$ nitric acid $(20 \mathrm{~mL})$ at $0{ }^{\circ} \mathrm{C}$ ice bath with stirring. After a week, the o-MCMB powder was collected by filtration and washed with deionized (DI) water until the solution $\mathrm{pH}$ reached 7.0

The morphology of graphite samples was examined using a field-emission scanning electron microscopy (FESEM, JEOL JSM$6700 \mathrm{~F})$ and a high-resolution transmission electron microscopy (HRTEM, JEOL JEM-3010). X-ray diffraction (XRD) patterns were recorded using a Bruker Advance-D8 with $\mathrm{Cu} \mathrm{K} \alpha$ radiation $(0.15406 \mathrm{~nm})$ at a scan rate of $1^{\circ} \mathrm{min}^{-1}$. Surface area and pore volume were analyzed from the $\mathrm{N}_{2}$ adsorption isotherms that were obtained with a gas adsorption analyzer (Micromeritics, ASAP 2010). The elemental analysis was made with an EA1110 (CE Instrument, Italy).

To prepare the composite electrodes, a mixture of e-MCMB powder, polytetrafluroethylene (PTFE), and carboxyl methyl cellulose binder (6:4 in weight ratio), and Super P (as the carbon additive for conductivity enhancement, 80:5:15 in weight ratio) was dispersed in DI water. The electrode composition was fixed at this ratio unless otherwise specified. The resulting slurry was spread on a piece of $\mathrm{Al}$ foil (thickness $=20 \mu \mathrm{m}$ ) that was used as the current collector and dried in vacuum at $120^{\circ} \mathrm{C}$ for $12 \mathrm{~h}$. The electrode was roll-pressed to enhance the interparticle contact and to ensure a better adhesion to the current collector.

The electrochemical characteristics (galvanostatic chargedischarge cycling and complex capacitance analysis) and thickness change of the e-MCMB electrode were examined as a half-cell to see its positive and negative activation behaviors, respectively. To this end, a counter electrode prepared with an activated carbon (BP20, $700 \mu \mathrm{m}$ thickness, surface area $=1800 \mathrm{~m}^{2} \mathrm{~g}^{-1}$, Kuraray Chemical Co.) and Li metal were used as the counter and reference electrode, respectively. Normally, $4 \mathrm{mg} \mathrm{cm}^{-2}$ of e-MCMB powder was loaded in the working electrode, and $40 \mathrm{mg} \mathrm{cm}^{-2}$ of BP20 powder in the counter electrode. Hence, the capacity of BP20/eMCMB cells tested in this work was limited by the working electrode (e-MCMB). The apparent area for both electrodes was $1.0 \mathrm{~cm}^{2}$. The electrolyte used was a mixture of tetraethyl ammonium tetrafluoroborate $\left(\mathrm{Et}_{4} \mathrm{NBF}_{4}, 0.95 \mathrm{M}\right)$ and lithium tetrafluoroborate $\left(\mathrm{LiBF}_{4}, 0.05 \mathrm{M}\right)$ that was dissolved in propylene carbonate. The macroscopic dimensional change of electrodes was traced using a homemade electrochemical dilatometer, the schematic drawing of which was provided in the previous reports. ${ }^{31-34}$ For this experiment, a disk-shaped e-MCMB composite electrode was positioned onto a disk of a stiff glass filter $(20 \mathrm{~mm}$ in diameter, $18 \mathrm{~mm}$ thick) and the counter electrode was located on the other side of the glass filter. As the dilatometer probe was positioned onto the surface of the working electrode, the observed height change represented only that of the working electrode. The applied force was about $3 \mathrm{~N}$, which is given by the weight of the probe. The change of interlayer spacing of the e-MCMB was traced using a homemade in situ XRD cell. ${ }^{35}$ A beryllium disk coated with aluminum (thickness $=250 \mathrm{~nm}$ ) was used as the current collector as well as X-ray win-

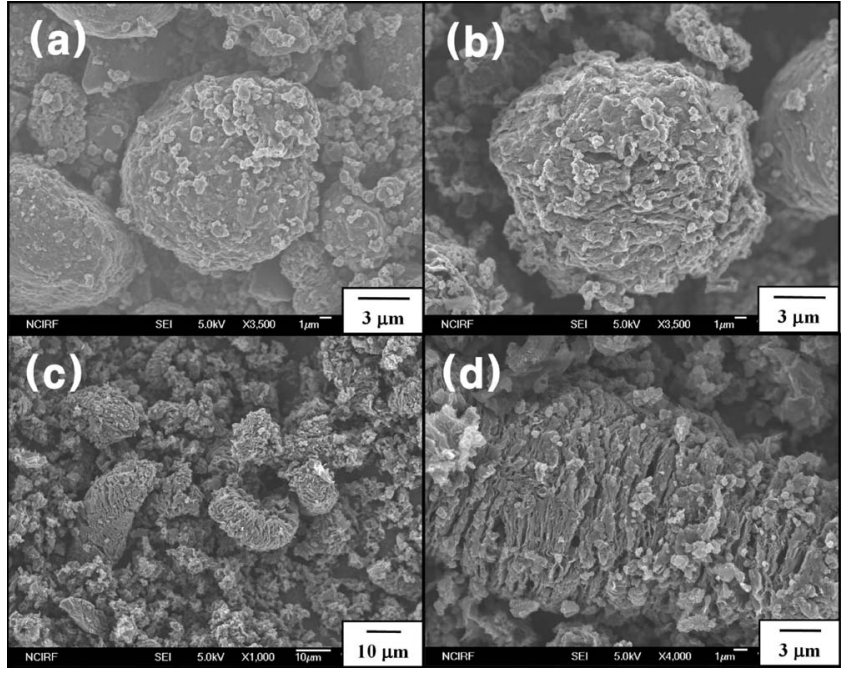

Figure 1. The FESEM images of graphite samples: (a) pristine MCMB, (b) o-MCMB, (c) e-MCMB, and (d) magnified view for e-MCMB.

dow. For the complex capacitance analysis, ac impedance measurements were made over the frequency range of $100 \mathrm{kHz}$ to $5 \mathrm{mHz}$ (Zahner, Im6e) with an ac amplitude of $5 \mathrm{mV}$.

The electrochemical characteristics (galvanostatic chargedischarge cycling) and thickness change of a full cell were also examined. To this end, two pieces of PP (polypropylene) separator were inserted between two identical e-MCMB electrodes, where the reference electrode ( $\mathrm{Li}$ foil) was inserted between two PP films. Galvanostatic charge-discharge cycling was done at a current density of $0.5 \mathrm{~mA} \mathrm{~cm} \mathrm{~m}^{-2}$ in the voltage range from 0.0 to $3.7 \mathrm{~V}$ with a WBC-3000 battery cycler (Xeno Co.). For the dilatometry experiment, the e-MCMB/e-MCMB full cell was placed onto the glass filter, such that the observed height change was the combined value of negative and positive electrodes.

\section{Results and Discussion}

Material characterization.- Figure 1 shows the FESEM images of pristine MCMB, o-MCMB, and e-MCMB. The morphologies of pristine MCMB (Fig. 1a) and o-MCMB (Fig. 1b) look similar to each other, but some cracks on the surface of o-MCMB can be recognized, indicative of a severe interlayer expansion as a result of addition of foreign elements into a graphene layer. The morphology of e-MCMB (Fig. 1c and d) is somewhat different from that of the former two. The sphere-shaped o-MCMB particles were transformed to wormlike ellipses in e-MCMB, the reason for which is not clearly identified. Simply, however, one can assume a deformation of carbon framework by the impetus of gaseous products $\left(\mathrm{CO}_{2}, \mathrm{SO}_{2}\right.$, and steam) that are generated during the heat-treatment of oxidized graphites. ${ }^{26,36}$ The interlayer expansion along with oxidation and heat-treatment can be estimated from the XRD patterns taken with these samples (Fig. 2). The pristine MCMB shows a strong (002) diffraction at $2 \theta=26.5^{\circ}$. The interlayer spacing $\left(d_{002}\right)$ is calculated to be $0.336 \mathrm{~nm}$ using the Bragg equation, which is very close to the value of natural graphites $(0.335 \mathrm{~nm})$. Upon oxidation, however, the (002) diffraction peak disappears while a new diffraction peak at $2 \theta=14^{\circ}$ develops, which corresponds to the (001) diffraction peak that is known for graphite oxides. ${ }^{36,37}$ The interlayer distance for $\mathrm{o}-\mathrm{MCMB}$ is calculated to be $0.636 \mathrm{~nm}$. When the o-MCMB is heattreated, the (002) diffraction peak is shifted back to the higher angle, but its intensity is far smaller than that of pristine MCMB, indicative of a decrease in crystallinity. The interlayer spacing of e-MCMB is now $0.404 \mathrm{~nm}\left(2 \theta=22.2^{\circ}\right)$. We call this material an expanded graphite because its interlayer distance is expanded as compared to that for the untreated MCMB. Table I compares the surface area, 


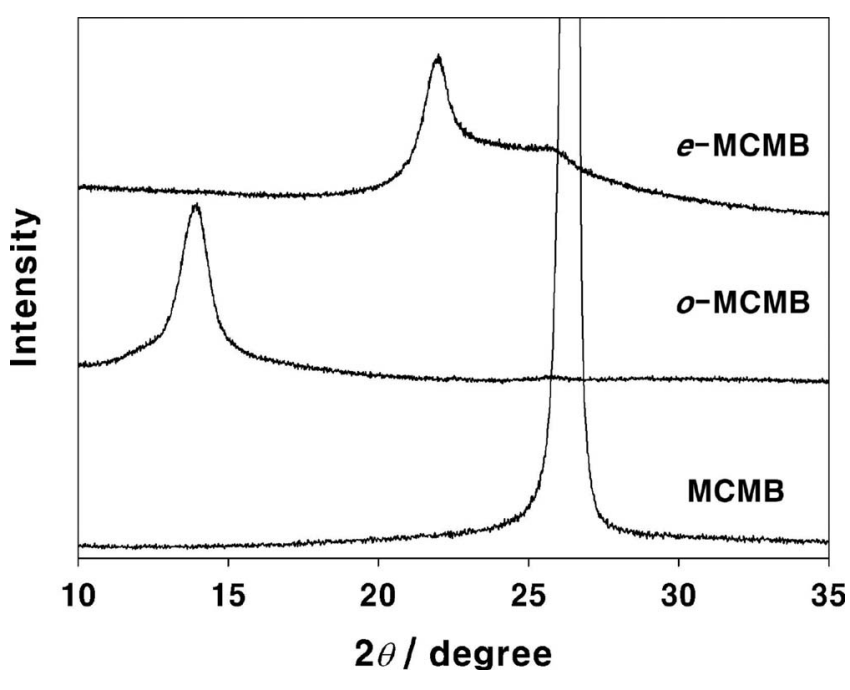

Figure 2. XRD patterns of MCMB, o-MCMB, and e-MCMB powders. Note the interlayer expansion in the e-MCMB powder.

pore volume, and elemental analysis data for three samples. Both the surface area and pore volume increase with oxidation, but decrease after the forthcoming heat-treatment to give the intermediate values. The surface area of e-MCMB is $21.8 \mathrm{~m}^{2} \mathrm{~g}^{-1}$, which is about 10fold larger than the pristine one but seems quite small if one considers a capacitor application. The amount of foreign elements that was represented by the $\mathrm{H} / \mathrm{C}$ and $\mathrm{O} / \mathrm{C}$ ratios was also increased upon oxidation but decreased after the heat-treatment. It is likely that the foreign elements that were introduced by oxidation were not completely removed even after the heat-treatment.

Electrochemical activation by positive polarization.-Figure 3 presents the half-cell characteristics of an e-MCMB electrode, where the e-MCMB electrode was tested as the positive electrode by potential cycling between the open-circuit voltage (ca. $3.0 \mathrm{~V}$ ) to 4.8 $\mathrm{V}\left(\mathrm{vs} \mathrm{Li} / \mathrm{Li}^{+}\right)$. In Fig. 3a, the galvanostatic charge-discharge voltage profile (solid line) is overlapped with the electrode height change (dotted line) traced with cycling. The result obtained in the first cycle is magnified in Fig. 3b. In both figures, the upward profile corresponds to the charging that is associated with the positive polarization of e-MCMB electrode and anion $\left(\mathrm{BF}_{4}^{-}\right)$accumulation, whereas the downward one corresponds to the discharging. At a glance, the electrode is swollen with charging, but contracted by discharging. The conversion point from swelling to contraction is well matched with the charge-discharge switching potentials (solid lines). An immediately apparent feature in Fig. 3 is that a voltage plateau appears at $4.6-4.8 \mathrm{~V}$ in the first charging but disappears from the second charging (Fig. 3a and b), implying that the charging process from the second cycle differs somewhat from that for the first cycle. The detailed discussion will be advanced in a later section.

Table I. Physico-chemical properties of pristine MCMB, $\mathrm{o}-\mathrm{MCMB}$, and e-MCMB.

\begin{tabular}{lcccccc} 
& \multicolumn{2}{c}{ Surface area and pore volume } & & \multicolumn{2}{c}{ Element analysis } \\
\cline { 2 - 3 } & $\mathrm{S}_{\mathrm{BET}} / \mathrm{m}^{2} \mathrm{~g}^{-1}$ & $\mathrm{~V}_{\text {total }} / \mathrm{cm}^{3} \mathrm{~g}^{-1}$ & & $\mathrm{H} / \mathrm{C}$ & $\mathrm{O} / \mathrm{C}$ \\
\hline MCMB & 2.11 & 0.02 & & - & 0.001 \\
o-MCMB & 33.1 & 0.10 & & 0.024 & 0.455 \\
e-MCMB & 21.8 & 0.06 & & 0.004 & 0.152
\end{tabular}

${ }^{a}$ Measured by Brunauer, Emmett, and Teller method from nitrogen adsorption isotherms.
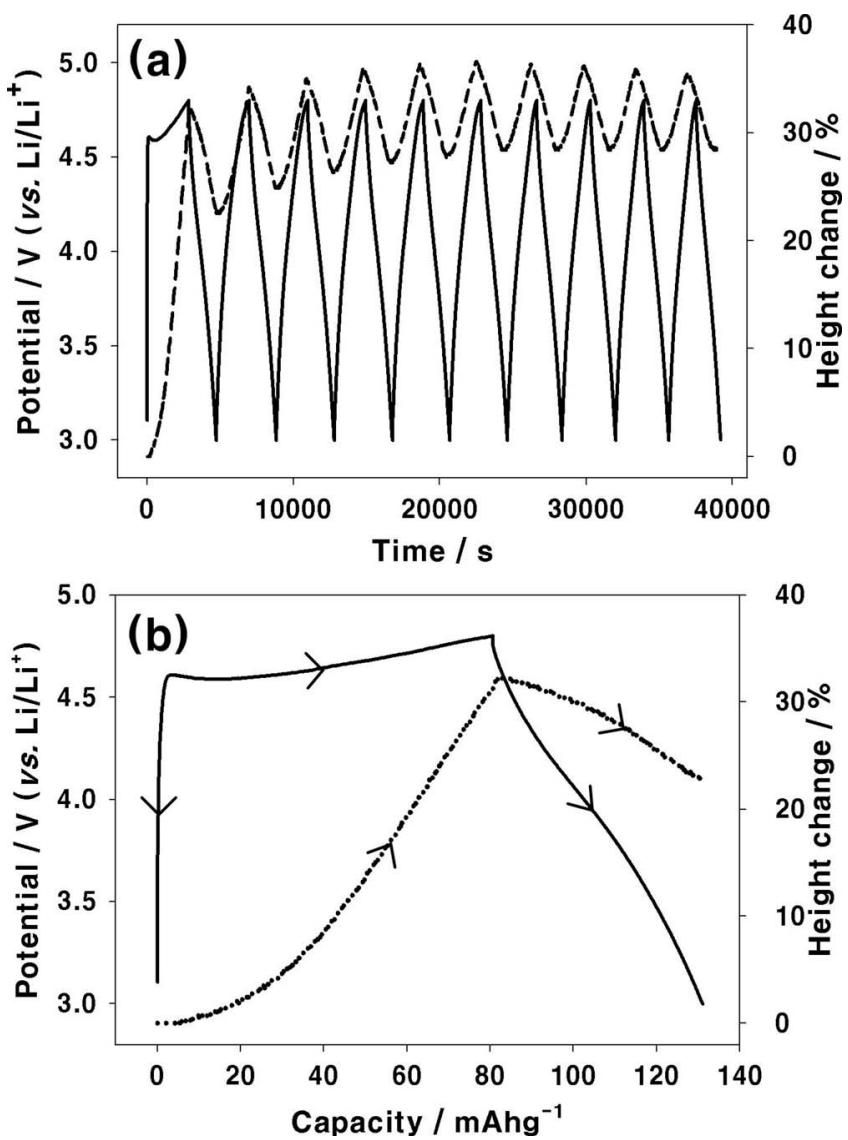

Figure 3. (a) The galvanostatic charge-discharge voltage profile (solid line) and electrode height change (dotted line) obtained with a half-cell cycling in the potential range of $3.0-4.8 \mathrm{~V}\left(\mathrm{vs} \mathrm{Li} / \mathrm{Li}^{+}\right)$. The open-circuit potential was $3.0 \mathrm{~V}$. (b) The magnified view for the first cycle. Note the presence of voltage plateau at $4.6-4.8 \mathrm{~V}$ in the first charging and concomitant electrode swelling at the same potential region. Also note that the voltage plateau is absent and the electrode swelling/contraction is only marginal (ca. 7\%) from the second cycle.

A close relationship between the voltage plateau appearing in the first charging and ion intercalation is evidenced by the electrode swelling that is occurring at exactly the same voltage range (4.6-4.8 V) where the plateau appears (Fig. 3b). The electrode height change amounting to $35 \%$ of the initial value in the first charging cannot be explained by any other processes except for interlayer expansion that is caused by ion $\left(\mathrm{BF}_{4}^{-}\right)$intercalation. That is, the other possible phenomena that may lead to a dimensional change of graphitic materials, $(i)$ enlarging of the intralayer $\mathrm{C}-\mathrm{C}$ bond length and (ii) an expansion of interlayer distance due to a decrease of surface tension with increasing excess charge in the double layer, are known to be far less significant as compared to ion intercalation. ${ }^{32}$

The assumption that the voltage plateau and electrode swelling evolved at 4.6-4.8 $\mathrm{V}$ are caused by ion intercalation has been validated by in situ XRD data. Figure 4a provides the microscopic change in the interlayer distance of e-MCMB particles. Also, a charge-discharge voltage profile (inset) is presented, wherein the points where the XRD data were taken are indicated as the numbers. When the electrode potential moves from the open-circuit potential $(3.0 \mathrm{~V})$ to the positive direction in the first charging, the XRD profile is not changed until the potential reaches the plateau. The (002) diffraction peak at $22.2^{\circ}$ is still alive (scan numbers 1 and 2). When the potential moves to the direction more positive than the plateau voltage (scan number 3 made at $4.8 \mathrm{~V}$ ), the peak at $22.2^{\circ}$ suddenly disappears, and never gains its intensity in the next cycles. A careful inspection of the XRD data, however, reveals the presence of a peak 

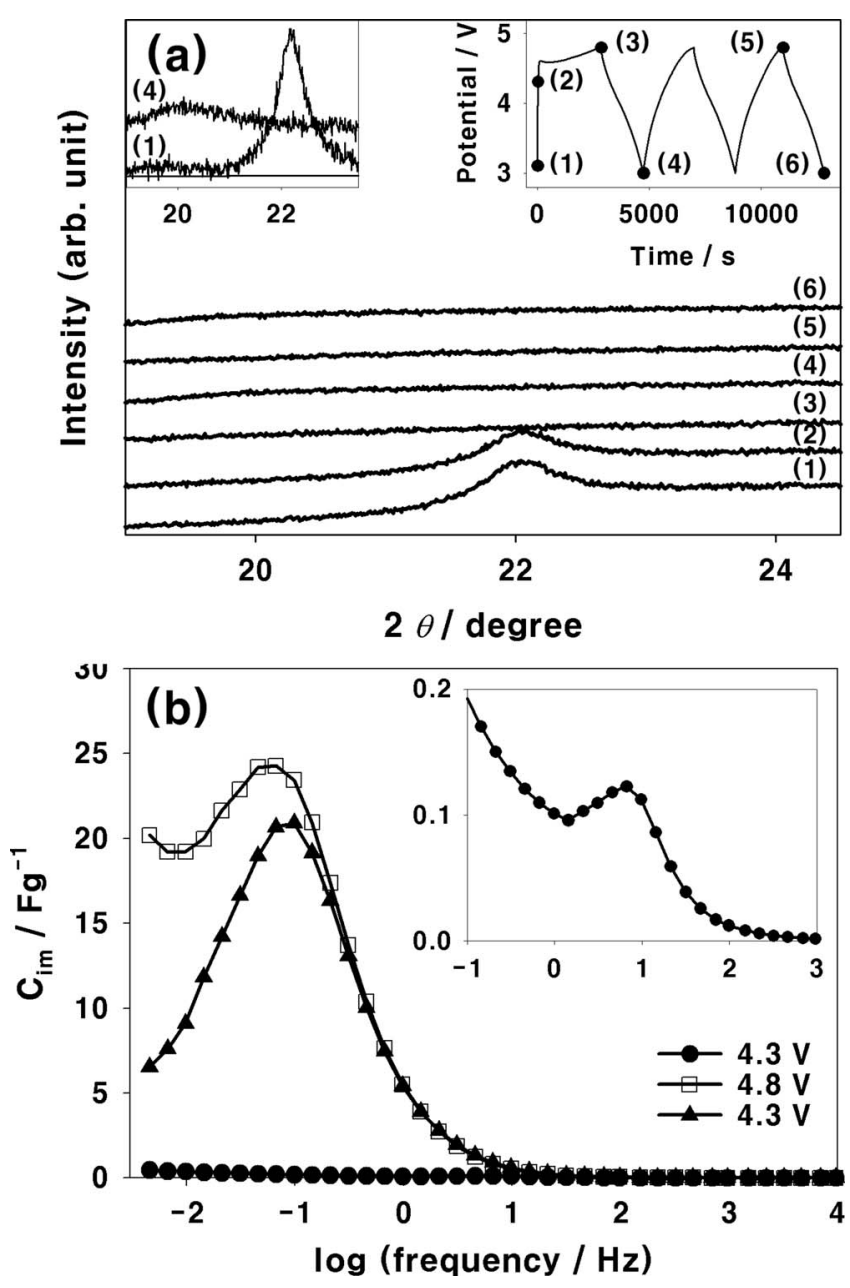

Figure 4. (a) The in situ XRD patterns of e-MCMB electrode with cycling. A voltage profile is provided in the inset, wherein the points where the XRD data were taken are indicated as the numbers. (b) The imaginary capacitance profiles obtained at three potentials; $4.3 \mathrm{~V}$ in advance, then $4.8 \mathrm{~V}$, and finally $4.3 \mathrm{~V}$ again after activation. Note a significant enlargement in the area of capacitive peak, indicative of a capacitance increase after electrochemical activation.

near $20^{\circ}$ that can barely be barely recognized (inset). These XRD data can account for the observations made with the dilatometry. First, if the peak at the lower angle $\left(20^{\circ}\right)$ is real, it implies a widening of the interlayer distance. Then, the macroscopic electrode height change occurring at the voltage plateau region can be ascribed to being caused by ion intercalation. The very weak diffraction peak at the lower angle $\left(20^{\circ}\right)$ can also be explained by the loss of layer ordering upon intercalation. Second, no restoration of the (002) peak to its original position supports that the interlayer remains widened after the first charging, which is in accordance with the dilatometry result.

To see if the interlayer expansion enlarges the ion-accessible surface area, thereby increasing the capacitance value of this electrode, the complex capacitance analysis was done. As reported earlier by us, the complex capacitance analysis can be a useful technique to assess the EDLC parameters, such as capacitance, rate capability, and degree of side reactions. ${ }^{38-41}$ In this analysis, impedance data are converted to complex capacitance and the imaginary part of complex capacitance is plotted as a function of frequency on a semilogarithmic scale (imaginary capacitance plots, $C_{\text {im }}$ vs $\log f$ ). In this plot, the capacitive component appears as a peak and the capacitance value is proportional to the area of this peak. The imaginary capacitance profiles taken with the e-MCMB electrode are rep- resented in Fig. 4b. With positive polarization, impedance measurements were made at $4.3 \mathrm{~V}$ in advance, then at $4.8 \mathrm{~V}$, and finally at $4.3 \mathrm{~V}$ again. The profile taken at $4.3 \mathrm{~V}$ initially shows a negligible size of capacitive peak, implying that the capacitance is very small. The profile has been magnified in the inset. When the electrode potential is raised up to $4.8 \mathrm{~V}$, a new peak appears at the lowerfrequency region with a notable increase in the peak area, indicative of an increase in the capacitance value. It is surprising that the e-MCMB electrode, which shows a negligible capacitance at $4.3 \mathrm{~V}$ initially (closed circles), delivers a much increased capacitance at the same voltage $(4.3 \mathrm{~V})$ after experiencing an interlayer expansion at $4.6-4.8 \mathrm{~V}$ (closed triangles). This phenomenon is termed as an electrochemical activation because the capacitance increases to a great extent after a charging (electrochemical reaction), and furthermore the enhanced capacitance is maintained thereafter.

Charge storage mechanism after electrochemical activation.The charge storage mechanism from the second cycle (after electrochemical activation) seems to be far different from that involved in the first charging (that is, intercalation) as hinted at by the following observations: the absence of voltage plateau, insignificant electrode swelling/contraction (ca. 7\%), and no restoration of interlayer distance to the original value. Even so, one cannot ensure that the charge storage is done by a simple adsorption because the following features are apparent. The electrode shows a repeated swelling/ contraction even if it is smaller (ca. 7\%) than that observed in the first charging. Moreover, the voltage profile is not linear but curved like those observed for pseudocapacitors. To straighten out this issue, the results obtained with a pristine MCMB and activated carbon (BP20, nongraphitic carbon) electrodes are provided. Figure 5a presents the charge-discharge voltage profile and electrode height change observed with the pristine MCMB electrode. Two features are immediately apparent. First, the voltage plateau appears in both charging and discharging in the first cycle, which is the case in the subsequent cycles. Second, the electrode swelling/contraction, which occurs at the same voltage range where the plateau evolves, is significant (ca. 100\%) in the first charging. More importantly, the height change amounts to $60-70 \%$ in the forthcoming cycles. Figure $5 \mathrm{~b}$ represents the in situ XRD data taken with this electrode. The (002) diffraction peak moves to the smaller angle $\left(25.7^{\circ}\right)$ upon first charging, but restores to the original value $\left(26.5^{\circ}\right)$ upon discharging. This reversible interlayer expansion/contraction also takes place in the next cycles. From these results, we can reach a conclusion that ion intercalation prevails not only in the first charging but also in the subsequent cycles in the pristine MCMB electrode.

By providing the results for untreated MCMB, it is now very likely that the charge storage in the electrochemically activated e-MCMB electrode is not proceeding by intercalation, but by adsorption. However, this assumption is not yet ensured because the e-MCMB electrode exhibits an appreciable height change (ca. 7\%) and the curved voltage profile. To clarify this, a voltage profile and electrode height change was measured for a BP20 electrode, wherein a simple adsorption is expected. As seen in Fig. 5c, the BP20 electrode also shows a nonstraight voltage profile but the height change amounts to only ca. $1 \%$. From this result, it may be safe to say that the charge storage mechanism in the electrochemically activated e-MCMB electrode is not a simple adsorption but a mixed adsorption/intercalation.

Figures $6 \mathrm{a}$ and $\mathrm{b}$ display the HRTEM images of e-MCMB electrode, whereas Fig. $6 \mathrm{c}$ and d shows those of the electrochemically activated one. It is seen that the slitlike pores are aligned in one direction in both samples (Fig. 6a and c). However, the slit width (that is, the interlayer distance) is not uniform with some deformation and the ordering is not perfect, which can account for the weak (002) diffraction peak for e-MCMB (Fig. 2 and 4a) and electrochemically activated e-MCMB (inset of Fig. 4a). It is difficult to estimate the interlayer distance from the magnified transmission electron microscopy images (Fig. 6b and d). At least, however, one can say that the interlayer distance in both samples is much smaller 

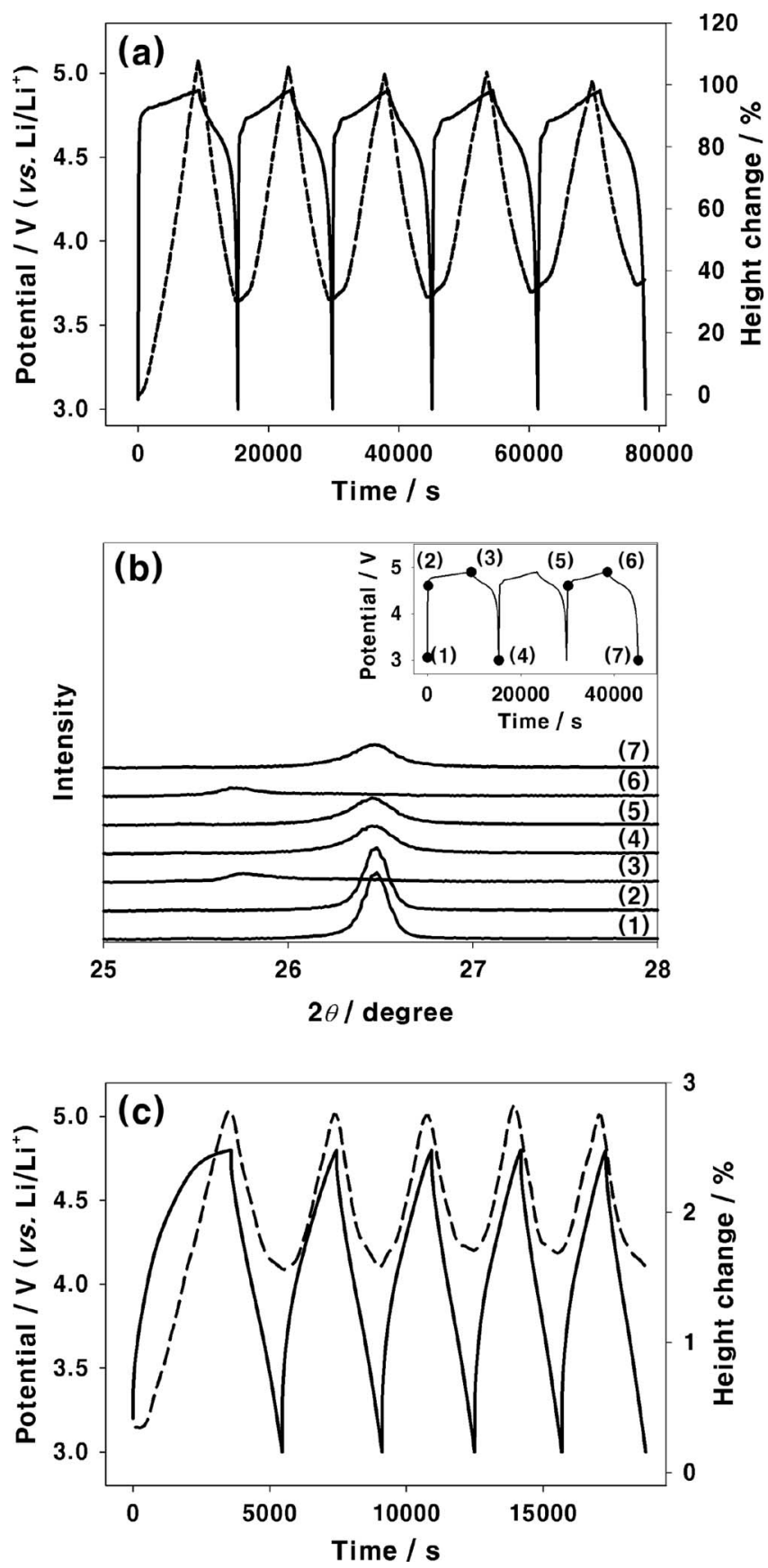

Figure 5. (a) The galvanostatic charge-discharge voltage profile (solid line) and electrode height change (dotted line) of pristine MCMB electrode obtained with a half-cell cycling in the potential range of $3.0-4.9 \mathrm{~V}\left(\mathrm{vs} \mathrm{Li} / \mathrm{Li}^{+}\right)$. (b) The in situ XRD patterns obtained with cycling. A voltage profile is provided in the inset wherein the points where the XRD data was taken are indicated as the numbers. (c) The galvanostatic charge-discharge voltage profile (solid line) and electrode height change (dotted line) of the BP20 electrode obtained with a half-cell cycling in the potential range of 3.0-4.9 V (vs Li/Li ${ }^{+}$.

than $1.0 \mathrm{~nm}$ but the interlayer gap becomes wider by electrochemical activation, which is roughly matched with those calculated from the XRD data; $0.404 \mathrm{~nm}\left(2 \theta=22.2^{\circ}\right)$ before activation and $0.45 \mathrm{~nm}$ $\left(2 \theta=20^{\circ}\right)$ after activation (Fig. 4a).

One curious but important observation here is that the electrochemically activated e-MCMB electrode delivers a high capacitance even if the interlayer distance is smaller than $1.0 \mathrm{~nm}$. Along this line, the recent reports made by Chmiola and his co-workers should

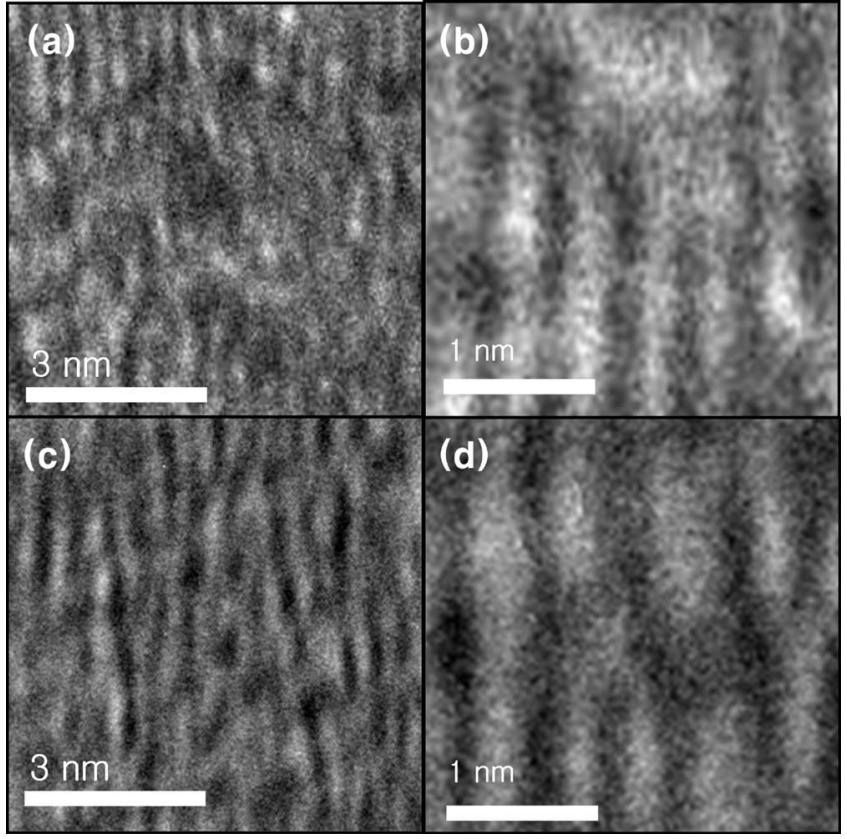

Figure 6. The HRTEM images of e-MCMB electrode before electrochemical activation [(a) and (b)] and after activation [(c) and (d)]. Note a slight widening of interlayer space by an electrochemical activation [(b) and (d)].

be mentioned. ${ }^{7,42}$ They observed an abnormally high capacitance with titanium carbide-derived carbon that has slit-shaped pores of less than $1.0 \mathrm{~nm}$, which is smaller than the size of solvated ions. To rationalize this, they proposed that ions enter the pores either bare or with partial solvent shells. The high capacitance observed with the electrochemically activated e-MCMB electrode in this work should also be explained by ion penetration either bare or with partial solvent shells. Furthermore, the same ion movement should take place in the electrochemical activation process because the interlayer distance amounts to only $0.404 \mathrm{~nm}$ for e-MCMB. That is, the electrochemical activation is propagated by ion intercalation rather than ion/solvent co-intercalation.

Electrochemical activation by negative polarization and full-cell test.- To see if the e-MCMB electrode is electrochemically activated by negative polarization, the charge-discharge cycling was done between the open-circuit voltage $(3.0 \mathrm{~V})$ and $1.0 \mathrm{~V}$. The voltage profile shown in Fig. 7a, more clearly in the magnified view for the first cycle in Fig. 7b, clearly shows that a voltage plateau appears at $1.3-1.0 \mathrm{~V}$ in the first charging, which is accompanied by an electrode swelling (ca. 30\%) in the same potential region. In addition, the e-MCMB electrode shows a similar activation behavior upon a negative polarization to that observed with the positive polarization. First, the voltage plateau never appears from the second cycle (Fig. 7a). Second, even if the e-MCMB electrode swells in the first charging up to ca. $30 \%$, it does not restore to the original dimension. Further, the electrode remains swollen in the subsequent cycles with an insignificant height change (ca. 10\%). The in situ XRD data obtained with charge-discharge cycling are presented in Fig. 8a, where it is seen that the (002) diffraction peak at $2 \theta=22.2^{\circ}$, which is intense before ion intercalation (scan numbers 1 and 2), suddenly disappears after a negative polarization down to $1.0 \mathrm{~V}$ (scan number $3)$. The barely identifiable peak at a lower angle $\left(2 \theta=20-21^{\circ}\right)$ reflects a widening of the interlayer distance after the first charging (inset). No return to the original diffraction angle in the next cycle reflects that the interlayer remains widened thereafter. All the observations made in Fig. 7 and 8a support that the e-MCMB electrode is electrochemically activated by a negative polarization, during which the interlayer is widened by cation intercalation, and remains wid- 

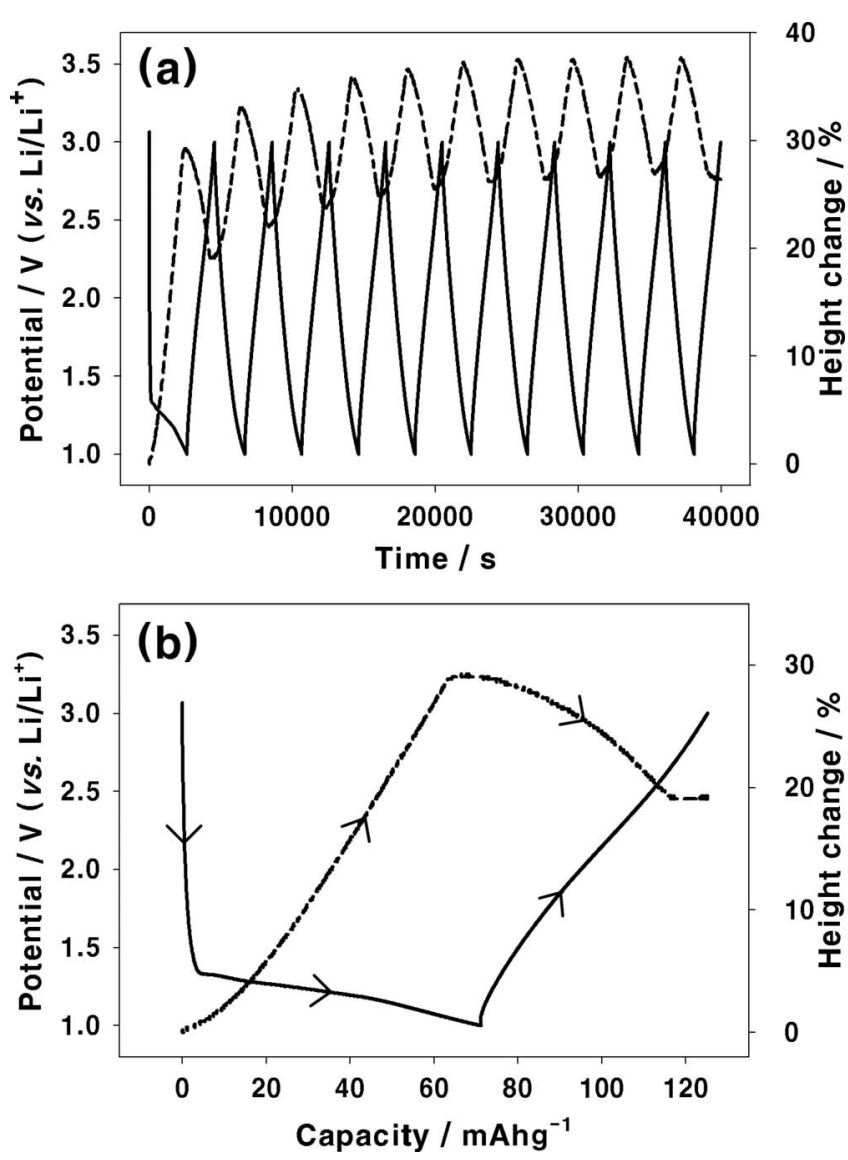

Figure 7. (a) The galvanostatic charge-discharge voltage profile (solid line) and electrode height change (dotted line) obtained with a half-cell cycling in the potential range of $3.0-1.0 \mathrm{~V}\left(\mathrm{vs} \mathrm{Li} / \mathrm{Li}^{+}\right)$. (b) The magnified view for the first cycle. Note the presence of voltage plateau at $1.3-1.0 \mathrm{~V}$ in the first charging and concomitant electrode swelling at the same potential region. Also note that the voltage plateau is absent and the electrode swelling/ contraction is only marginal (ca. 10\%) from the second cycle.

ened thereafter. A capacitance increase by such an electrochemical activation can further be ascertained from the impedance data shown in Fig. $8 \mathrm{~b}$, which were obtained at $1.7 \mathrm{~V}$ in advance, then at $1.0 \mathrm{~V}$, and finally at $1.7 \mathrm{~V}$ again. Before activation, the electrode shows a negligible capacitance as evidenced by the negligible size of the capacitive peak (in the inset). A notable increase in the capacitance is, however, confirmed by the area of the capacitive peak (the profile obtained at $1.0 \mathrm{~V}$ ). The fact that the enlarged capacitance is maintained thereafter (that is, it is electrochemically activated) can also be confirmed from the imaginary capacitance profile obtained at 1.7 $\mathrm{V}$ after the activation (closed triangles).

The finding that the e-MCMB electrode can be electrochemically activated by both positive and negative polarization encouraged us to fabricate a full cell with two identical e-MCMB electrodes. Figure $9 \mathrm{a}$ shows the charge-discharge voltage profile and electrode height change recorded with cell cycling in the voltage range of $0.0-3.7 \mathrm{~V}$. A voltage plateau appears at $3.6 \mathrm{~V}$ in the first charging, which must be related with ion intercalation in both electrodes. Also note that the voltage plateau never appears in the subsequent cycles. The full cell is swollen in the first charging, not contracted to the initial dimension, but rather damps within a 10\% range (dotted line). This is what was observed with the half-cells. Figure $9 \mathrm{~b}$ shows the potential profiles for the positive (solid line) and negative electrode (dotted one) taken with cell cycling. Before cycling, both electrodes remain at the open-circuit potential $(3.0 \mathrm{~V})$. During the first charging, the positive electrode shows a voltage plateau at 4.6-4.8 V, whereas the negative electrode plateaus at $1.3-1.1 \mathrm{~V}$ in accordance
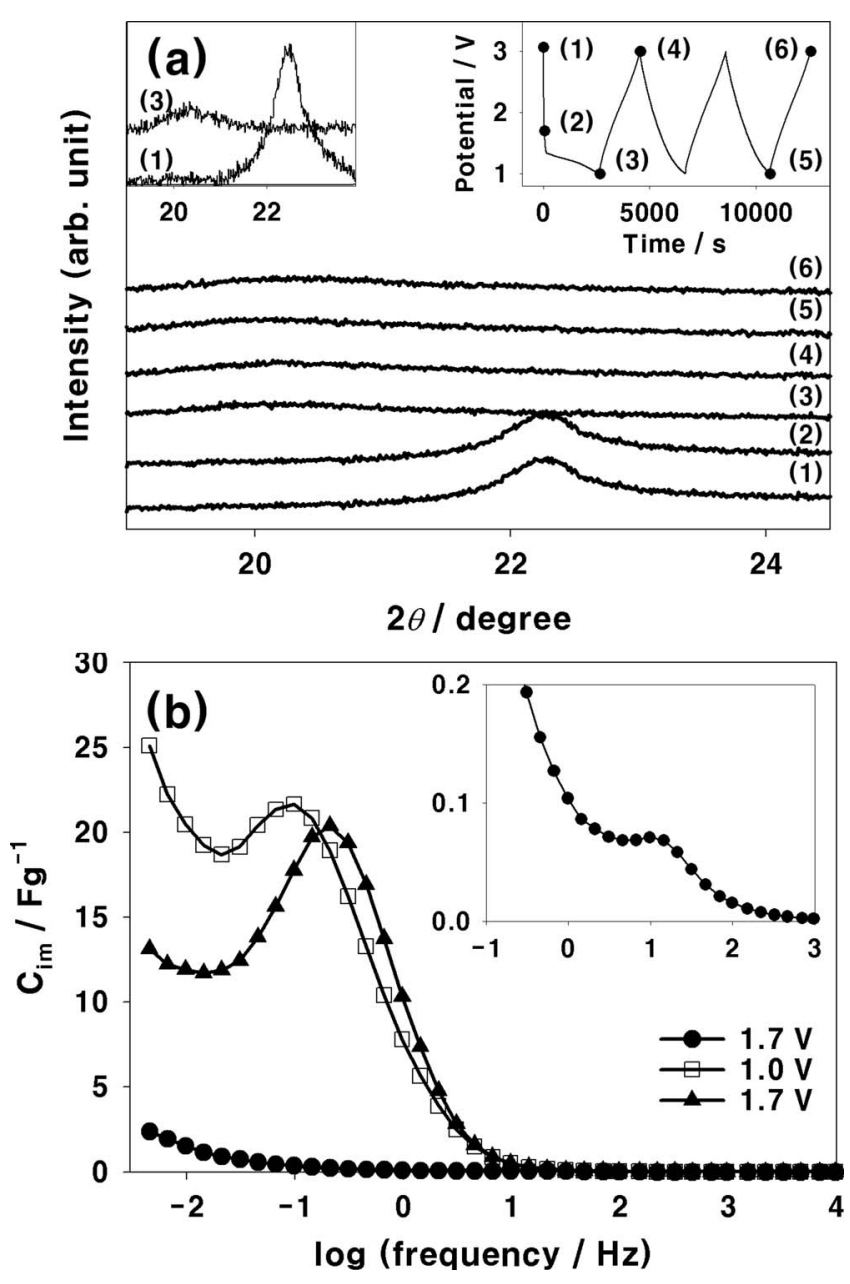

Figure 8. (a) The in situ XRD patterns of e-MCMB electrode with cycling. A voltage profile is provided in the inset, wherein the points where the XRD data were taken are indicated as the numbers. (b) The imaginary capacitance profiles obtained at three potentials; $1.7 \mathrm{~V}$ in advance, then $1.0 \mathrm{~V}$, and finally $1.7 \mathrm{~V}$ again after activation. Note a significant enlargement in the area of capacitive peak, indicative of a capacitance increase after electrochemical activation.

with the results obtained with the half-cell tests. From the second cycle, the cell shows a symmetric voltage profile without a voltage plateau (Fig. 9b). Figure 9c presents the capacitance of full-cell and coulombic efficiency obtained by galvanostatic charge-discharge cycling. The volume-specific capacitance that was calculated on the basis of the volume of two dry e-MCMB electrodes was $30 \mathrm{~F} \mathrm{~mL}^{-1}$ in the first cycle, which is slightly decreased to $24 \mathrm{~F} \mathrm{~mL}^{-1}$ after 100 cycles. This capacitance fading may be caused by several reasons, but not identified in this work. At such a high working voltage $(3.7 \mathrm{~V})$, however, a decomposition of the electrolyte and electrode itself can be readily imagined. The relatively high population of foreign elements in e-MCMB (Table I) may be associated with electrode degradation under such an extremely high electrode polarization. The coulombic efficiency is somewhat low initially, which seems to be due to faradaic reaction associated with electrolyte decomposition. After that, however, the cell shows a coulombic efficiency of $>99 \%$. The volume-specific capacitance $\left(24-30 \mathrm{~F} \mathrm{~mL}^{-1}\right)$ and working voltage $(3.7 \mathrm{~V})$ of this EDLC are comparable to those for the Nanogate capacitor.

There are two final points to address. The first point is the huge amount of electrode swelling in the activation process, $30-35 \%$ as shown in Fig. 3a and 7a. Such an electrode swelling cannot be explained by the volume expansion of e-MCMB particles by them- 

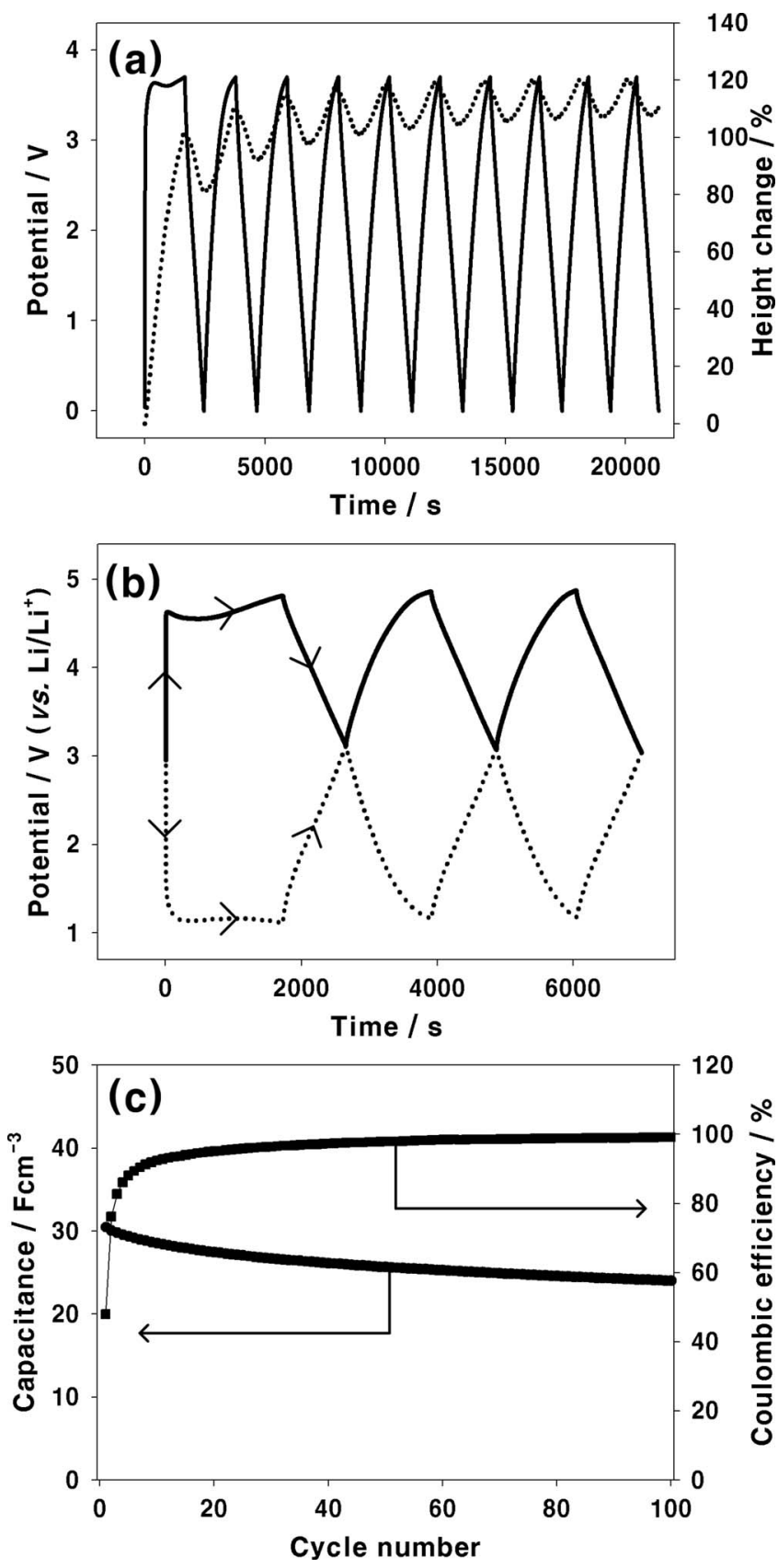

Figure 9. (a) The galvanostatic charge-discharge voltage profile (solid line) and electrode height change (dotted line) obtained with a full cell in the voltage range of 0.0-3.7 V. (b) The potential variation of the positive (solid line) and negative (dotted line) electrode in the full cell fabricated with two e-MCMB electrodes and Li metal reference electrode. (c) The cycle performance of the full cell obtained at a current density of $0.5 \mathrm{~mA} \mathrm{~cm}$. For this experiment, the electrode was prepared from the mixture of e-MCMB:PTFE:Super P (86:7:7 in weight ratio).

selves. The interlayer expansion upon electrochemical activation is only $11 \%$, from 0.404 to $0.45 \mathrm{~nm}$. The excessive electrode swelling may be associated with a loosening in the electrode packing (between e-MCMB particles, carbon additive, and polymer binder) that is caused by volume expansion of e-MCMB particles. The second concern is the prerequisite for electrochemical activation. The intercalation of cations (for instance, $\mathrm{Et}_{4} \mathrm{~N}^{+}$) and anions $\left(\mathrm{BF}_{4}^{-}\right.$) into interlayer of graphitic carbons has been reported previously. ${ }^{20-25,43-47}$ On the basis of the charge-discharge cycling and dilatometric behaviors, the graphitic carbons can be divided into two groups: pris- tine graphites and expanded graphites. The highly crystalline natural and artificial graphites, and graphitizable carbons belong to the former group, whereas the $\mathrm{KOH}-$ or $\mathrm{K}_{2} \mathrm{CO}_{3}$-activated graphites, expanded needle cokes, ${ }^{30}$ and e-MCMB studied in this work can be categorized into the latter group. The common observation with the former is that ion intercalation, which takes place over a certain onset potential, is accompanied by a voltage plateau, widening of interlayer distance, and electrode swelling. The most apparent difference from the latter group is that all these events take place in every cycle. ${ }^{43-47}$ That is, as seen in Fig. $5 \mathrm{a}$ and $\mathrm{b}$ in this work, the swollen electrode by charging (that is, intercalation) is contracted back to the original dimension upon discharging, and this swelling/ contraction in a full range is repeated in the continuing cycles. This illustrates that the intercalation process in the former group does not generate new ion-accessible sites that can be utilized permanently. Rather, the interlayer sites are instantly utilized for charge storage with ion intercalation in each cycle. Hence, the intercalation occurring in the former group cannot be categorized as an electrochemical activation if one defines this process as a generation of ionaccessible sites by a single intercalation process, and the asgenerated sites are permanently utilized for ion accumulation. A common feature for the latter group is that the interlayer distance is substantially expanded as compared to that for the highly crystalline graphites $\left(d_{002}=0.335-0.34 \mathrm{~nm}\right)$. The $d_{002}$ value is $0.36-0.385 \mathrm{~nm}$ for the $\mathrm{KOH}$-activated graphitizable carbons for the Nanogate capacitor $^{20,21}$ and $0.404 \mathrm{~nm}$ for the e-MCMB in this work. It is thus concluded that the prerequisite for electrochemical activation is the interlayer expansion by any treatments. As far as the authors know, two methods have been utilized for this purpose in the literature: chemical activation (Nanogate capacitor) and heat-treatment of oxidized graphitic carbons as presented in this work.

\section{Conclusion}

This work provides evidence that the expanded graphite is electrochemically activated both by positive and negative polarization. When the e-MCMB electrode is polarized over a certain onset potential in both the positive and negative direction, ion-accessible sites are generated with an interlayer expansion that is induced by ion intercalation. The presence of a voltage plateau that is associated with intercalation was confirmed from the charge-discharge voltage profiles. The microscopic change of the interlayer distance and concomitant macroscopic dimensional change of the electrode were ascertained by the in situ XRD and electrochemical dilatometry study, respectively. Additional points of value are summarized as follows:

1. The interlayer spacing of e-MCMB is larger than that for untreated $\mathrm{MCMB}$, thereby an easier ion intercalation can be assumed.

2. The electrochemical activation was accomplished by ion intercalation (not by ion/solvent co-intercalation) in the first charging period. Once activated, the interlayer of e-MCMB remains widened, allowing the newly generated sites to be easily accessed by ions. Even so, the charge storage in the electrochemically activated e-MCMB electrode is not made by a simple ion adsorption, but by a mixed adsorption/intercalation. Given the fact that untreated graphites cannot be electrochemically activated, that is, ion intercalation does not create new sites, it is concluded that the prerequisite for electrochemical activation is the expansion of interlayer distance in graphitic carbons.

3. The major electrode swelling takes place only in the electrochemical activation period, but the electrode swelling/contraction is marginal after the activation. This feature is practically important because a repeated swelling/contraction in a wider dimension frequently destructs the electrode integrity.

4. The electrochemically activated e-MCMB electrode shows a promising capacitor behavior as a full cell. The capacitance is as high as $24-30 \mathrm{~F} \mathrm{~mL}^{-1}$ and the working voltage amounts to $3.7 \mathrm{~V}$ with a charge-discharge coulombic efficiency of $>99 \%$. 


\section{Acknowledgment}

This work was supported by KOSEF via the Research Center for Energy Conversion and Storage.

Seoul National University assisted in meeting the publication costs of this

\section{References}

1. A. Nishino, J. Power Sources, 60, 137 (1996).

2. B. E. Conway, Electrochemical Supercapacitors, Kluwer Academic/Plenum Publishers, New York (1999).

3. A. Burke, J. Power Sources, 91, 37 (2000).

4. R. Kötz and M. Carlen, Electrochim. Acta, 45, 2483 (2000).

5. E. Frackowiak and F. Beguin, Carbon, 39, 937 (2001).

6. S.-R. Hwang and H. Teng, J. Electrochem. Soc., 149, A591 (2002).

7. J. Chmiola, G. Yushin, Y. Gogotsi, C. Portet, P. Simon, and P. L. Taberna, Science, 313, $1760(2006)$

8. H. Nakagawa, A. Shudo, and K. Miura, J. Electrochem. Soc., 147, 38 (2000)

9. H. Teng, Y. Chang, and C. Hsieh, Carbon, 39, 1981 (2001).

10. M. Endo, T. Maeda, T. Takeda, Y. J. Kim, K. Koshida, H. Hara, and M. S. Dresselhaus, J. Electrochem. Soc., 148, A910 (2001).

11. D. Lozano-Castello, M. A. Lillo-Rodenas, D. Cazorla-Amoros, and A. LinaresSolano, Carbon, 39, 741 (2001).

12. Y. Guo, H. Zhang, N. Tao, J. Qi, Z. Wang, and H. Xu, Mater. Chem. Phys., 82, 107 (2003).

13. H. Tamai, M. Kunihiro, M. Morita, and H. Yasuda, J. Mater. Sci., 40, 3703 (2005).

14. T. Morimoto, K. Hiratsuka, Y. Sanada, and K. Kurihara, J. Power Sources, 60, 239 (1996).

15. K. Edström, A. M. Andersson, A. Bishop, L. Fransson, J. Lindgren, and A. Hussénius, J. Power Sources, 97-98, 87 (2001).

16. O. Barbieri, M. Hahn, A. Herzog, and R. Kötz, Carbon, 43, 1303 (2005).

17. G. G. Amatucci, F. Badway, A. D. Pasquier, and T. Zheng, J. Electrochem. Soc., 148, A930 (2001).

18. T. Brousse, R. Marchand, P.-L. Taberna, and P. Simon, J. Power Sources, 158, 571 (2006).

19. X. Hu, Y. Huai, Z. Lin, J. Suo, and Z. Deng, J. Electrochem. Soc., 154, A1026 (2007).

20. M. Takeuchi, K. Koike, T. Maruyama, A. Mogami, and M. Okamura, Denki Kagaku oyobi Kogyo Butsuri Kagaku, 66, 1311 (1998)
21. M. Takeuchi, T. Maruyama, K. Koike, A. Mogami, T. Oyama, and H. Kobayashi, Electrochemistry (Tokyo, Jpn.), 6, 487 (2001).

22. S. Mitani, S.-I. Lee, K. Saito, S.-H. Yoon, Y. Korai, and I. Mochida, J. Power Sources, 133, 298 (2004).

23. S. Mitani, S.-I. Lee, K. Saito, S.-H. Yoon, Y. Korai, and I. Mochida, Carbon, 43 $2960(2005)$

24. S. Mitani, S.-I. Lee, K. Saito, Y. Korai, and I. Mochida, Electrochim. Acta, 51, 5487 (2006).

25. T. Aida, I. Murayama, K. Yamada, and M. Morita, J. Power Sources, 166, 462 (2007).

26. E. Matuyama, J. Phys. Chem., 58, 215 (1954).

27. T. Nakajima and Y. Matsuo, Carbon, 32, 469 (1994).

28. A. Hamwi and V. Marchand, J. Phys. Chem. Solids, 57, 867 (1996).

29. Y. Matsuo and Y. Sugie, Carbon, 36, 301 (1998).

30. I.-J. Kim, S. Yang, M.-J. Jeon, S.-I. Moon, H.-S. Kim, Y.-P. Lee, K.-H. An, and Y. H. Lee, J. Power Sources, 173, 621 (2007).

31. M. Hahn, O. Barbieri, R. Gallay, and R. Kötz, Carbon, 44, 2523 (2006).

32. M. Hahn, O. Barbieri, F. P. Campana, R. Kötz, and R. Gallay, Appl. Phys. A: Mater Sci. Process., 82, 633 (2006).

33. S. Park, T. Kim, and S. M. Oh, Electrochem. Solid-State Lett., 10, A142 (2007).

34. T. Kim, S. Park, and S. M. Oh, J. Electrochem. Soc., 154, A1112 (2007)

35. P. W. Ruch, M. Hahn, F. Rosciano, M. Holzapfel, H. Kaiser, W. Scheifele, B Schmitt, P. Novák, R. Kötz, and A. Wokaun, Electrochim. Acta, 53, 1074 (2007)

36. Y. Matsuo, T. Niwa, and Y. Sugie, Carbon, 37, 897 (1999).

37. S. Yang, H. Song, and X. Chen, Carbon, 44, 730 (2006).

38. J. H. Jang and S. M. Oh, J. Electrochem. Soc., 151, A571 (2004).

39. S. Yoon, J. H. Jang, B. H. Ka, and S. M. Oh, Electrochim. Acta, 50, 2255 (2005).

40. J. H. Jang, S. Yoon, B. H. Ka, Y. H. Jung, and S. M. Oh, J. Electrochem. Soc., 152 A1418 (2005).

41. B. H. Ka, S. Yoon, and S. M. Oh, J. Korean Electrochem. Soc, 10, 252 (2007)

42. J. Chmiola, C. Largeot, P. L. Taberna, P. Simon, and Y. Gogotsi, Angew. Chem., Int Ed., 47, 1 (2008).

43. M. Yoshio, H. Nakamura, and H. Wang, Electrochem. Solid-State Lett., 9, A561 (2006).

44. H. Wang and M. Yoshio, Electrochem. Commun., 8, 1481 (2006).

45. F. P. Campana, M. Hahn, A. Foelske, P. Ruch, R. Kötz, and H. Siegenthaler, Electrochem. Commun., 8, 1363 (2006).

46. L. J. Hardwick, M. Hahn, P. Ruch, M. Holzapfel, W. Scheifele, H. Buqa, F Krumeich, P. Novák, and R. Kötz, Electrochim. Acta, 52, 675 (2006).

47. H. Wang, M. Yoshio, A.-K. Thapa, and H. Nakamura, J. Power Sources, 169, 375 (2007). 